\title{
Combining Skeletal Poses for 3D Human Model Generation using Multiple Kinects
}

\author{
Kevin Desai, Balakrishnan Prabhakaran \\ The University of Texas at Dallas \\ Richardson, Texas, USA \\ \{Kevin.Desai,bprabhakaran\}@utdallas.edu
}

\author{
Suraj Raghuraman \\ Mobiweb Inc \\ Plano, Texas, USA \\ suraj@mobiwebinc.com
}

\begin{abstract}
RGB-D cameras, such as the Microsoft Kinect, provide us with the 3D information, color and depth, associated with the scene. Interactive 3D Tele-Immersion (i3DTI) systems use such RGB-D cameras to capture the person present in the scene in order to collaborate with other remote users and interact with the virtual objects present in the environment. Using a single camera, it becomes difficult to estimate an accurate skeletal pose and complete 3D model of the person, especially when the person is not in the complete view of the camera. With multiple cameras, even with partial views, it is possible to get a more accurate estimate of the skeleton of the person leading to a better and complete 3D model. In this paper, we present a real-time skeletal pose identification approach that leverages on the inaccurate skeletons of the individual Kinects, and provides a combined optimized skeleton. We estimate the Probability of an Accurate Joint (PAJ) for each joint from all of the Kinect skeletons. We determine the correct direction of the person and assign the correct joint sides for each skeleton. We then use a greedy consensus approach to combine the highly probable and accurate joints to estimate the combined skeleton. Using the individual skeletons, we segment the point clouds from all the cameras. We use the already computed PAJ values to obtain the Probability of an Accurate Bone (PAB). The individual point clouds are then combined one segment after another using the calculated $\mathrm{PAB}$ values. The generated combined point cloud is a complete and accurate $3 \mathrm{D}$ representation of the person present in the scene. We validate our estimated skeleton against two well-known methods by computing the error distance between the best view Kinect skeleton and the estimated skeleton. An exhaustive analysis is performed by using around 500000 skeletal frames in total, captured using 7 users and 7 cameras. Visual analysis is performed by checking whether the estimated skeleton is completely present within the human model. We also develop a 3D Holo-Bubble game to showcase the real-time performance of the combined skeleton and point cloud. Our results show that our method performs better than the state-of-the-art approaches that use multiple Kinects, in terms of objective error, visual quality and real-time user performance.
\end{abstract}

Permission to make digital or hard copies of all or part of this work for personal or classroom use is granted without fee provided that copies are not made or distributed for profit or commercial advantage and that copies bear this notice and the full citation on the first page. Copyrights for components of this work owned by others than ACM must be honored. Abstracting with credit is permitted. To copy otherwise, or republish, to post on servers or to redistribute to lists, requires prior specific permission and/or a fee. Request permissions from permissions@acm.org.

MMSys'18, fune 12-15, 2018, Amsterdam, Netherlands

(c) 2018 Association for Computing Machinery.

ACM ISBN 978-1-4503-5192-8/18/06 .. \$15.00

https://doi.org/10.1145/3204949.3204958

\section{CCS CONCEPTS}

- Information systems $\rightarrow$ Multimedia information systems; - Computing methodologies $\rightarrow$ Reconstruction; Mixed / augmented reality; $3 D$ imaging; Tracking; Point-based models; • Software and its engineering $\rightarrow$ Interactive games;

\section{KEYWORDS}

Interactive 3D Tele-Immersion, 3D Model, Combined Skeleton, Point Cloud Combination

\section{ACM Reference Format:}

Kevin Desai, Balakrishnan Prabhakaran and Suraj Raghuraman. 2018. Combining Skeletal Poses for 3D Human Model Generation using Multiple Kinects. In MMSys'18: 9th ACM Multimedia Systems Conference, fune 1215, 2018, Amsterdam, Netherlands. ACM, New York, NY, USA, 12 pages. https://doi.org/10.1145/3204949.3204958

\section{INTRODUCTION}

Generation of a complete and accurate 3D model of the person is required in various 3D Tele-Immersion (3DTI) applications such as Exergames for Rehabilitation [7], Virtual Laboratory training [8], Musculoskeletal Examination [23], various 3D games, etc. Using a single Kinect camera, only one side of the person can be captured in real time. Different methods exist that can generate a combined 3D model over time using only one camera, such as Kinect Fusion [12], Fusion4d [10], etc. Such methods, though accurate to an extent, don't work in real time and also need manual interventions. In order to capture the complete 3D model of the person in real time, multiple cameras are required. In addition to visually seeing the 3D human model, i3DTI systems [9] also need the person to interact with virtual objects present in the scene. For such interaction purposes, estimating the human skeleton is necessary. In this paper, we propose an efficient human pose estimation strategy that generates an accurate skeleton using multiple Kinect cameras. Using the combined skeleton, we propose a fast and accurate registration method to obtain a combined 3D point cloud model of the person.

Human pose estimation has various applications in activity recognition, natural user interface, gait analysis, etc. Various marker based methods exist, which capture the accurate human pose. Such methods are invasive in nature because of the use of extra markers attached to the body. These methods though extremely accurate, require special setup, are expensive and take a lot of time, making them useless for real-time applications such as 3D Tele-Immersion (3DTI) [14]. Marker-less pose estimation has been made possible with the advent of multi-view and RGB-D depth cameras. RGB-D cameras, with their extra depth stream, allow us to get an accurate estimation of the person's pose in real time without the use of any markers. Microsoft's Kinect [6] is one such RGB-D camera that 


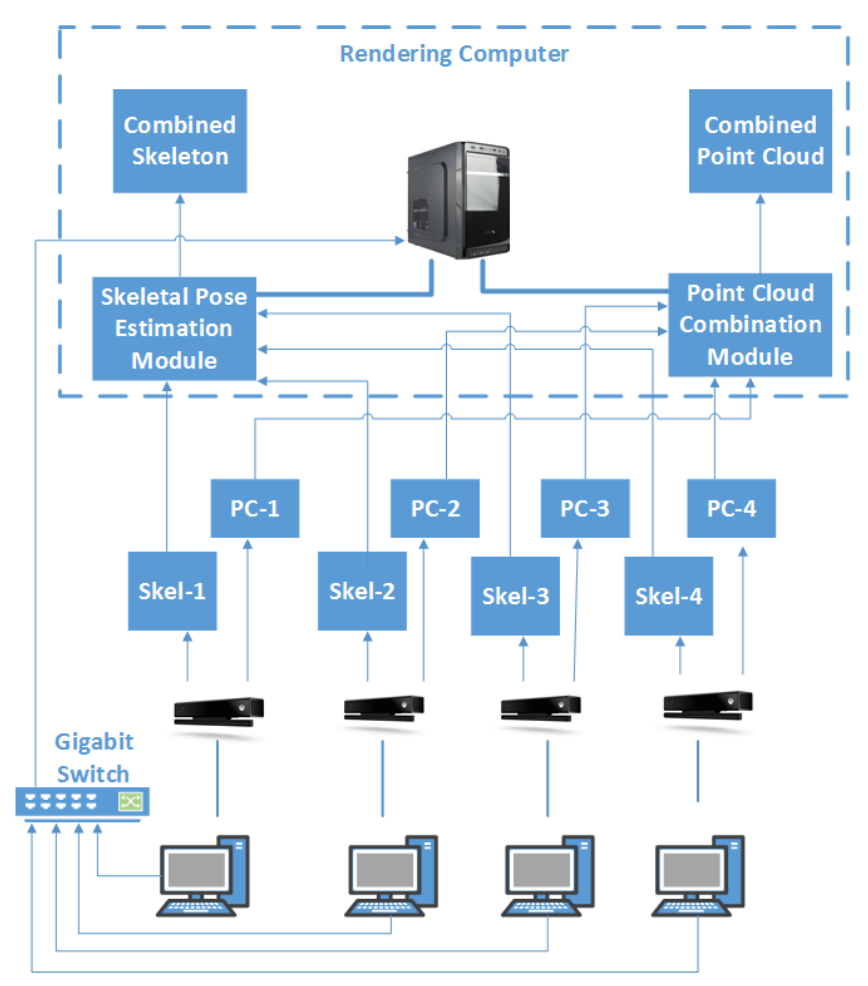

Figure 1: Overview of our system: An example using 4 Kinect cameras.

provides the functionality to estimate human pose in the form of a skeleton [21]. The Kinect has been used in variety of applications that require human skeleton estimation. Majority of the existing methods for human pose estimation mainly use a single Kinect camera. Using only one camera restricts the person to always face in the direction of the camera, in order to obtain an accurate skeleton. However, such skeleton estimations can quickly become inaccurate mainly due to sensor noise, occlusion or when only a part of the human body is visible. Section 3 covers the single Kinect skeleton challenges in detail. Use of multiple cameras to capture the same scene allows us to get more data about the person as well as the pose. In this paper, we use the skeletal data from multiple Kinects and combine it to obtain a better skeletal pose estimation.

\subsection{Contributions}

In this paper, we propose a system that uses multiple Kinect data and performs the following tasks in real time:

- Skeletal pose estimation is performed by combining the skeleton data obtained from multiple Kinect cameras. The combined skeleton has better accuracy and is robust to the challenges observed by individual skeletons.

- Combined 3D model is generated which provides a complete $3 \mathrm{D}$ view of the person in real-time. The combined skeleton is used to combine the point cloud data from individual Kinects to obtain clean, accurate and complete 3D point cloud model of the person.

\subsection{Proposed Approach}

The proposed system consists of two major parts:

- Skeletal pose estimation is performed by combining all individual Kinect skeletons. A probability based consensus approach is used to estimate the accurate combined skeleton. We assume that the individual joint estimations from the Kinect are more reliable than the complete skeleton and we evaluate them to determine the Probability of an Accurate Joint (PAJ). Then a distance constrained greedy consensus approach is used to select joints, such that the joint PAJ of the set is the maximum. The direction of the person is then used to determine the right side of the body from the left side. We determine the direction of the person using the direction of feet or torso. If those two fail, we use the knee curvature to determine the direction. Doing so ensures that all of the joints are labeled correctly, irrespective of which direction the person is facing. While it is possible to use face tracking to determine the direction that a person is looking, it is not necessarily the same direction in which that person's body is aimed. Section 4 provides detailed steps involved in obtaining the combined and optimized skeleton.

- Point cloud combination is performed by using the above estimated skeleton and the individual Kinect point clouds. First, we segment the individual point clouds into different bones using the individual skeletons. We then use the PAJ for each skeleton joint to obtain the Probability of an Accurate Bone (PAB). The PAB values from all Kinects are used to combine the individual point clouds one segment after another. Overlapping points from different cameras are eliminated or combined based on the PAB values associated with them. We also take into consideration the Kinect joint estimation accuracy in deciding whether the segment from one camera is to be used or not. Based on this, the combined Point Cloud is obtained which is an accurate 3D model estimation of the real world person. Section 5 explains the point cloud combination process in detail.

Figure 1 shows the overview of our system. The example uses 4 cameras, but the system can be used with any number of Kinect cameras. Each Kinect is connected to an individual machine which are in turn connected to a rendering computer using a gigabit switch. The skeleton and point cloud extracted from each Kinect are passed into a skeletal pose estimation module and a point cloud combination module respectively. At the end, we obtain a combined optimized skeleton and a combined 3D point cloud model of the person.

Figure 2 shows one pose made by a person along with the skeleton and combined point cloud generated by our method. The combined skeleton is evaluated against two state-of-the-art methods. A distance based error score is calculated by comparing the estimated skeleton against the skeleton from the Kinect which has the best view of the person. Visual analysis is performed by checking that the estimated skeleton is always inside the person, irrespective of the camera viewing angle or the pose. The combined skeleton is also visually compared against the individual Kinect skeletons. We also create a 3D Holo-Bubble game that forces the users to move around the entire scene to burst the virtual bubbles using their 


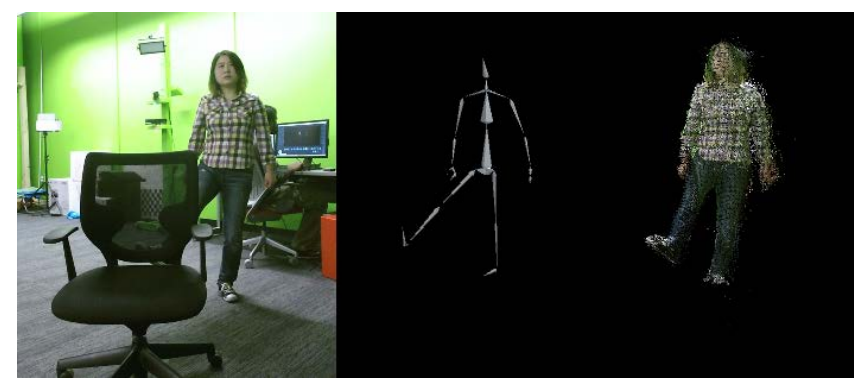

Figure 2: Our approach on a person present in the capture scene occluded by a chair in one camera. Left to Right: Color image, estimated skeleton and combined point cloud.

skeleton. The game showcases the real-time performance of the combined skeleton and point cloud as well as the improvement in user performance. Results obtained from the analyses show our method to be performing much faster and at the same time has more accuracy for obtaining the combined skeleton and the 3D model.

\section{RELATED WORK}

Human pose identification is a widely researched topic in the field of graphics and computer vision. Various methods exist which extract pose from 2D images. Here, we are only focused on skeletal pose estimation from RGB-D cameras, specifically Kinect. Microsoft provides the Kinect SDK [15] which provides a skeleton. The Kinect extracts local features from the depth image, and classifies them using a random forest to determine the skeleton [21]. A temporal probabilistic model is suggested by [27], to overcome certain types of occlusion using prior estimations, joint constraint, and inter-frame velocity. Both of these methods are machine learning approaches that require millions of poses for training to achieve good results. These methods work really great with applications that require the subject to be seen completely within the range and facing the camera. Since both of them are single skeleton approaches, they fail when there is occlusion.

Since single Kinect methods are prone to the problems mentioned in Section 3, lot of research is being performed in combining skeleton information from multiple cameras. A novel ellipsoidal skeleton is designed by [22] such that the geometry detail of the tracked object can be well captured. The approach is very accurate and robust which deals with occlusion issues. The proposed motion tracking method only uses the depth information of the tracked object and is formulated as a pure energy optimization, without any other modalities of data or training data needed. This method generates a very high quality skeleton but it takes a significant amount of time making it unsuitable for real-time applications. Three Kinect cameras are used by [1] to predict the human motion in occluded settings. They use randomly sampled candidate positions and maximize an energy function to infer the joint positions. They define a joint confidence value based on depth and postures, and use fuzzy inference to model sensor noise. Duplex Kinect setup, with two cameras kept 90 degrees apart, is used by [25] to determine the skeleton of the person, even when occluded from the view of one of the Kinect cameras. They minimize the joint position differences in the two cameras by using bone length as a hard constraint and the trade-off between inconsistent joint positions as a soft constraint. The approach overcomes the varying bone length problem but it can't adapt to the joint position jitter. Also, it is limited to only 2 Kinects and hence the complete view of the person is not obtained.

[13] uses multiple Kinect cameras with one as the reference for other cameras to transfer their coordinates into a common space. They use a simple averaging strategy to combine the joint position values from multiple Kinect skeletons by giving same weight to each camera. Because of that, if all the 3 skeleton joint positions are inaccurate, the final skeleton will also be inaccurate. [11] also uses a similar averaging strategy but selects points that have high confidence and are close to each other. [4] extends this averaging strategy by making it generic and applicable to any multi-view systems that use skeletal data. Also, there is no need to manually determine which camera gives the best view of the scene making it more suitable for real world tasks. [16] uses a similar weighted averaging strategy to fuse the skeletons into one. They consider the scenario that when two cameras are placed face-to-face, the skeleton seen from both the cameras have their left and right joint assignments flipped. They check for this situation by estimating the relative distance between the original and flipped skeleton. Using this strategy they flip the left and right side of the skeleton. However, they don't have a robust way of identifying the front or the back and hence the final skeleton obtained can also have its left and right flipped.

Majority of the works in literature, for generating a combined 3D model of a person, focus on reconstructing a dense surface. Of the real-time systems that exist, Kinect Fusion [12] and Fusion4d [10] do a great job in reconstructing the entire 3D model of the person in real time using a single Kinect camera. However, these methods don't just focus on the person but the entire scene. At the same time, these methods need manual intervention and movement of the camera or the capture object in a specific manner, making it invasive to a certain extent. Our focus here is to not generate the underlying surface but generate a complete $3 \mathrm{D}$ point cloud representation of just the person in the scene. Different 3D meshing strategies can be applied on top of the obtained point cloud to generate the 3D surface model. Hence, our problem reduces to that of a merging problem rather than capture. Among the various methods that exist in combining point clouds, Iterative Closest Point (ICP) algorithm is the most widely used producing the best results. [20] shows the different variants of ICP that have been proposed.

[5] proposed a method of estimating human pose using a combined point cloud generated from multiple Kinect cameras. They combine the point clouds using a simple ICP strategy to obtain a combined 3D model of the person. They change the view such that the face of the person is in front, and then estimate the skeleton based on the projected depth image. [26] proposed a similar architecture where they combine the point clouds from multiple cameras and then compute the human skeleton. They down-sample the depth image by a factor of 16 and then segment the person out of that. The extracted point cloud from all the cameras are merged in the global space to obtain the resultant joint point cloud. They compute their own shape model and generate a new skeleton based on this combined point cloud. However, they only consider two cameras, front and back but don't consider the extra points 


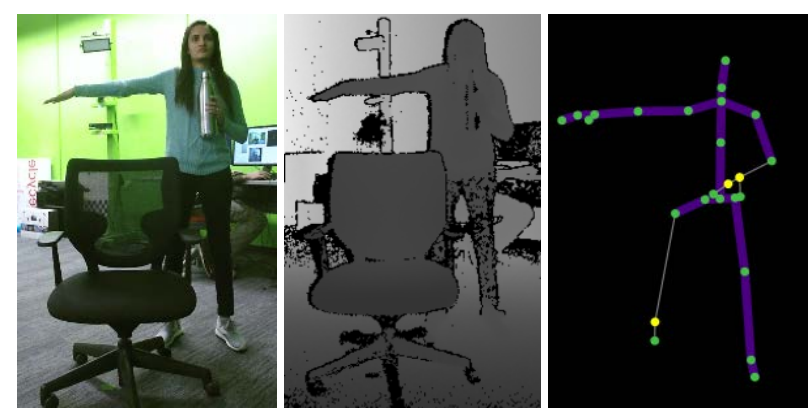

Figure 3: Challenges from Single Kinect Skeleton

that would be added in the final joint point cloud if the cameras have partial overlapping views. Both methods combine the entire human point cloud using ICP or direct merge. They don't take into account the inaccuracies existing in the original point cloud while combining them. This results in inaccurate, noisy and dense point clouds.

\section{SINGLE KINECT SKELETON CHALLENGES}

Single frame single camera skeleton identification of the Kinect is largely accurate, but it is susceptible to many problems in certain situations. In order to better understand the challenges faced, we group them based on the source as follows:

- Sensor Noise : Kinect V2 sensors use time of flight to measure the depth at each pixel. This measurement is influenced by lighting, reflectiveness of the object, dust, curvature etc. The depth sensor has a relatively low resolution of $512 \times 424$ pixels with a wide angle lens. As a result, depending on the location of the person (at least $1 \mathrm{~m}$ from the camera to capture entire person), the number of pixels to encode the person is relatively low. Hence, regions like the hand and fingers are not captured with a great level of detail.

- Occlusion : For accurate skeleton identification, a direct line of sight between the camera and the different parts of the body are necessary. These line of sight problems, or occlusion, can be categorized as external and self-occlusion. External occlusion occurs when an external object occludes the object that is being tracked. Self-occlusion occurs when deformation or the pose of the object hides certain other parts of the object. For example, when a person stands with their hands behind their back, the body blocks the view of the hand.

- Object Interference : Position of objects, even if not occluding the view of the camera, plays a key role in the detected skeleton. For example, if a person is sitting in a chair then the detected skeleton incorporates the chair as well, resulting in an inaccurate skeleton. Even in an area with no external objects, the ground interferes with the detection of the leg, especially the knee and the foot.

- Jitter : Since Kinect uses time of flight, the depth image obtained is very noisy. Each frame of depth image has varying depth values even without any change in the capture space. From this noisy depth image, the skeleton is estimated [21].
Kevin Desai, Balakrishnan Prabhakaran and Suraj Raghuraman

The resultant skeleton is noisy and jitters a lot even without any movement.

- Motion Blur : When actions are performed very swiftly, there is a noticeable difference in the alignment between the depth and skeleton data. This particular problem might be due to the difference in the frame used for skeleton estimation and the depth information captured, or just the sensor noise.

- Fitting : The skeleton is an estimate of the pose of the person, and should ideally be located in the middle of the body. Since only one camera view is available, the skeleton may be too close to the surface or too far behind the person. It was noticed that Kinect detected skeletons are often times too far away from the surface of the body.

- Lighting : The lighting and reflectiveness of the surfaces surrounding the person have significant influence on the overall skeleton that is detected. The Kinect sensor has issues in estimating the depth of shiny black surfaces. Because of this, the location of the head and shoulder region is prone to errors when capturing the person from behind.

Figure 3 shows the different skeleton challenges observed using a single Kinect camera. While some issues like object interference, lighting, and sensor noise (to some extent), can be reduced by managing the capture area appropriately, other issues like motion blur and edge noise from the sensor, can only be resolved with better sensors or sensor specific filtering approaches. Fitting errors are largely caused by partial viewing angles and occlusion. In our 3DTI setup, we reduce depth image errors by using non-reflective carpet and controlled lighting. We use a temporal smoothing approach [3] to reduce the skeleton jitter, explained in Section 4. A set of calibrated Kinects is used to reduce the level of occlusion significantly for a large number of poses.

\section{SKELETAL POSE ESTIMATION}

Kinect was designed more from a natural user interface point of view, hence, it assumes that the user will be facing the camera while performing all of the activities. The sensor provides color, depth and skeleton estimates for the captured scene. The skeleton estimate is generated based on just the depth image captured by that particular sensor. The sensor captures depth information using a time of flight approach, resulting in large occlusions for poses where the person is not directly facing the camera. It uses a depth variation based interest point selector and descriptor to allow tagging of individual parts of the depth image. A random forest based classifier is trained with millions of real and synthetic depth images, of different sized users performing varying activities. Using these classifiers, various parts of the body are tagged and then connected to generate the final human skeleton. We believe that the Kinect sensor approach, though proprietary, has a very high accuracy, especially when it comes to detecting the location of parts of the body for nonoccluded poses. This can primarily be attributed to the huge training data used to train the classifier. Implementation of the classifier on the GPU allows skeleton estimation in real time, which allows us to estimate [18], animate [17] or even interact [7] with the 3DTI systems more naturally. Hence, instead of capturing a large training 

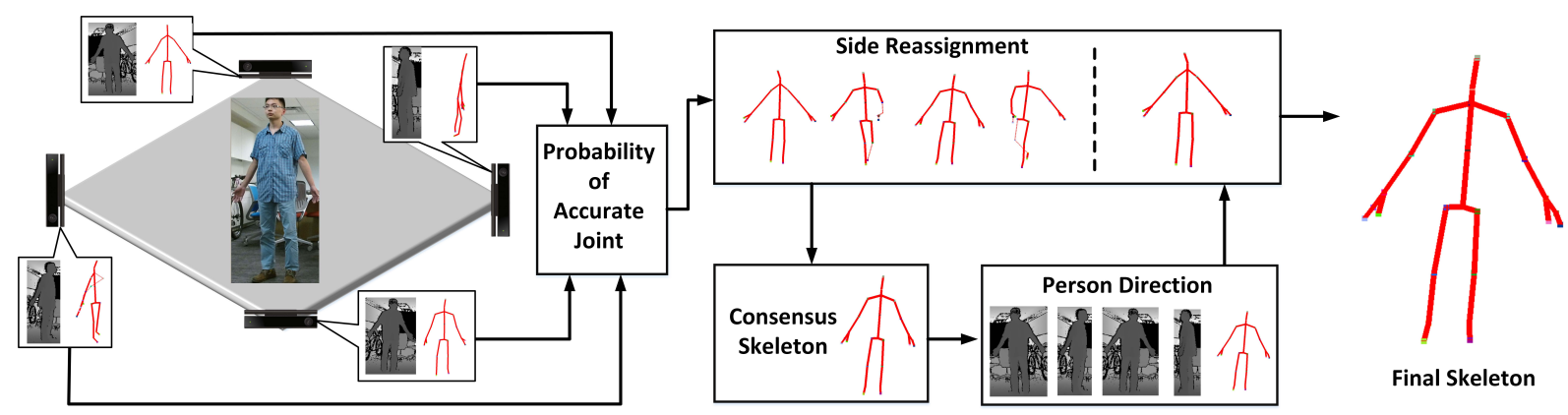

Figure 4: Our skeletal pose estimation approach.

data set with multiple depth cameras, we leverage our approach on the accuracy of body part detection of the Kinect.

Our skeletal pose estimation approach uses the skeleton information from multiple Kinects to generate a single skeleton of the person in the scene. Following points highlight the important steps involved in our method:

- Instead of relying on the entire skeleton, which might be prone to errors due to various factors (Section 3), we treat the skeleton joints independent of each other. Treating these joints separately ensures that our method only uses the primary classification results of the Kinect approach, as described in [21].

- Since the Kinect skeleton has the problem of jittery joint positions, we pre-process the original Kinect skeleton by applying a temporal smoothing on the joints, as described in Microsoft's white paper [3]. The smoothing filter used is based on the Holt Double Exponential Smoothing method used for statistical analysis of economic data. This provides smoothing with less latency than other smoothing filter algorithms.

- Even though the Kinect provides joint state information, indicating whether the joint is tracked, inferred or not tracked, in our experiment we found this information to be very unreliable. We decided to only use the joints whose joint state is tracked, according to the Kinect.

- We then calculate the Probability of an Accurate Joint (PAJ) estimation by the Kinect, based on the joint position in the local Kinect depth image, and the observed trends during experimentation.

- After calculating the PAJ of these tracked joints, we transform all the joints from the various Kinects to a single 3D space. We determine the direction of the person using feet or torso direction. If the information for the above is unreliable we use knee curvature to find the person's direction. This direction information is used to assign the side of the joints correctly.

- Once all the joint sides are re-assigned, we use a distance constrained consensus approach that maximizes the overall PAJ, to determine the location of the joints in 3D space.

The entire skeletal pose estimation method is shown in Figure 4.

\subsection{Probability of Accurate Joint}

When each Kinect only has a partial view of the person, it is highly likely that none of the Kinects are going to return a complete and accurate skeleton. Hence, picking a skeleton based only on the point of view is not feasible. Treating the skeleton as a hierarchical set of joints allows us to use all the estimated skeletons appropriately to form a single accurate skeleton of the person. We consider all joints to be independent of each other but order them based on hierarchy, which allows us to process the root joint first, and the terminal joints at the end. Various independent factors influence the accuracy of the joint. Each of these factors can be equated to a probability, which can in turn be combined together to provide the Probability of an Accurate Joint (PAJ). The key factors we are referring to are the following:

- Skeleton Orientation : The Kinect skeleton is most accurate when the person is directly facing the camera. This is because of the extremely large training set used for the skeleton estimation. Hence, while combining the skeletons, we assign higher priority to the skeleton obtained by the camera towards which the person is facing. We assign lower priority to the skeleton of the cameras capturing the person 90 degrees from the side. Since the Kinect does not differentiate between the front and the back of the person, the skeleton from the camera exactly behind the person is considered with the same probability as the skeleton from the camera exactly in front of it. Thus, the PAJ based on the skeleton orientation w.r.t. camera is $P(S)=\frac{\Pi / 2-\left|\tan ^{-1} \frac{R . x}{R . z}\right|+C A}{\Pi / 2}$. Here, $C A$ is the camera angle pre-calculated based on the global $3 \mathrm{D}$ co-ordinate system and the extrinsic camera calibration and is in the range $[0, \Pi / 2] . R$ is the direction of the skeleton i.e. the normal of the plane obtained from 3 different skeleton joints. Depending on the original joint detection accuracy, we take either of the two planes - one made by hip-center, shoulder-left, shoulder-right or another made by hip-left, hip-right, shoulder-center.

- Joint Position : The depth estimations of the Kinect sensor are accurate only in a fixed region [24]. If the joint is present outside this region, the probability that the joint is accurately estimated diminishes as it keeps moving away from the region. So, the PAJ based on position $P(J)=\left(\left|\tan ^{-1} \frac{x}{z}\right|-\right.$ $\left.\tau_{x}\right) *\left(\left|\tan ^{-1} \frac{y}{z}\right|-\tau_{y}\right) *\left(z-\tau_{z}\right)$. Here, $\tau$ is the threshold distance selected that provides the best skeleton recognition 
results. Based on [24] and the scene setup, $\tau$ is set to be $(1.25,1.05,3.0)$.

- Bone Angle : The angle of the bone to the capture plane of the camera seems to play a vital role in determining the position of the joints. The more acute that the angle between the plane and the bone is, the more likely that the joint has been estimated correctly. Based on this, we estimate that the probability of the joint being empirically identified due to the bone angle is calculated as $P(A)=|\cos \theta||\cos \phi|$, where $\theta$ is the angle between the bone corresponding to the joint and the $\mathrm{xz}$ plane, and $\phi$ is the angle between the bone and yz plane.

- Bone Length : We know that the person's skeleton is of a fixed size, so there should be little to no variation in the person's bone size from frame to frame. The length of the bone between 2 consecutive skeletons, from the same camera, has to be almost identical for it to be a valid joint. Based on this, we can estimate the probability of the joint based on bone length variation $P(B)=1-\frac{\left|L_{i}-L_{i-1}\right|}{\max \left(L_{i}, L_{i-1}\right)} \sigma$ : where $L_{i}$ is length of the bone in the current frame, $L_{i-1}$ is the length of the bone in previous frame, and $\sigma$ is the scale factor that is set to 5 , allowing up to $20 \%$ variation.

All probability calculations are bound in $[0,1]$ to ensure that there is no overflow. By combining all the conditional probabilities of these factors, we can estimate the overall probability of an accurate joint. Since the factors are independent of each other, $P A J=P(S) P(J) P(A) P(B)$. For all the joints, and all the detected skeletons from the cameras, we compute the PAJ.

\subsection{Person Direction \& Side Assignment}

The Kinect skeleton uses local features to determine the position of the joints and as a result, the entire direction of the person is lost. Hence, the Kinect skeleton tracker assumes that the person is facing the camera. This assumption is not true in our multiple Kinect camera setup, since cameras surround the person in order to get a complete view of him/her. Some cameras capture the person from behind, creating issues with the way that the left and right side of the body joints are tagged by the Kinect. To determine the person's direction, we can't just rely on the direction of the head because the person can turn his/her head and look in different direction. We can use the direction of the vector passing through the point of intersection of the feet and bisecting the angle between them, as the direction of the person. If the feet are parallel to each other, then the direction is same as the feet. Unfortunately, due to ground noise, the Kinect does not accurately identify the feet. Usually the person's direction is same as the torso direction. By using the hip and shoulder joint positions, it is possible to get the axis of the torso region by fitting a plane through these points and using the normal information. We use the disparity in the size of the front and rear part of the head to determine the direction of the person on the axis. As shown in Figure 5, even when a person turns their head, the front part of the head always protrudes outward from the neck or the body.

The feet direction is determined to be correct or not, using the associated joint confidence. As mentioned above, the foot joint is not determined accurately by Kinect. Hence, if the foot joint

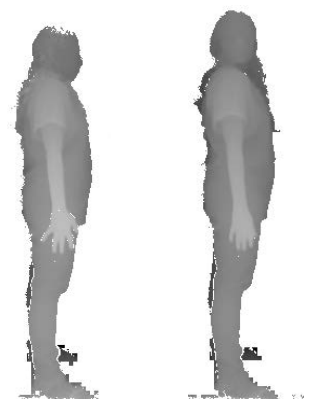

Figure 5: Person direction estimation using the head and torso region: The front part of the head protrudes outwards more than the back, irrespective of looking forward (left) or sideways (right).

confidence from the Kinect $<0.5$, we move to the Head and Torso based direction. We use the disparity in the size of the front and rear part of the head to determine the head direction. If the detected human head disparity is less than or equal to one, the direction of the head cannot be obtained accurately. In such situations, we study the knee curvature in the depth image to estimate knee direction. Due to limited degree of freedom, muscles around the knee region form a concave side (rear part) and a convex side (front part). To estimate the curvature, we select eight points around the knee orthogonal to the bone. Then we further sample another eight points using a Fast detector like pattern [19]. To avoid interference from noise, we use a wide selector that selects a single point in a $16 \times 16$ neighborhood. Each point is then placed around the original point to form a $3 \times 3$ monge patch. Then, only the curvature of the center point in the patch is estimated. A Monge patch is a patch $\mathbf{x}: U \rightarrow R^{3}$ of the form $\mathbf{x}(u, v)=\left[\begin{array}{lll}u & v & f(u, v)\end{array}\right]^{T}$ where $U$ is an open set in $R^{2}$ and $f: U \rightarrow R$ is a differentiable function. Corresponding Gaussian curvature is computed as:

$$
K=\frac{f_{u u} f_{v v}-f_{u v}^{2}}{\left(1+f_{u}^{2}+f_{v}^{2}\right)^{2}}
$$

where the subscript denotes the partial differentiation $f_{u}=\frac{\partial f}{\partial u}$. The entire computation can be performed on GPU for faster performance. The curvature value at each point indicates a saddle point of 0 , whereas it is a concave surface if greater than 0 and convex if less than 0 . Using this information, we select the two opposing points, one concave and one convex with high magnitudes, and use the convex point as the direction of the leg. If required, a side fill approach can be used at the knee to determine the size of the feet, if they are not visible.

Side Assignment Procedure : Once we have all the joints and we know the direction of the person, we can assign the correct sides to each skeletal joint. Using the hip center joint for reference, we start tagging the joints to the right of the hip center as the right hip and right shoulder, and the left side as the left hip and left shoulder. After the torso region joints have the sides assigned, the side information is propagated to all the child joints, like the knee, elbow, wrist, ankle, etc. This side assignment strategy ensures that crossed arms or legs are tagged accurately. In the case of a single Kinect, since the joints are tagged based on individual proximity 
with respect to the camera view, the same joint can be considered right or left, depending on the location of the camera. Even after transforming all of the skeletons to the same primary camera point of view using the extrinsic calibrations, the sides can still be incorrectly assigned. So to ensure the correct marking of joint sides, after the skeletons are transformed to the primary camera view, the joints are reassigned using the procedure mentioned above.

\subsection{Combined Skeleton}

To determine the skeleton from the various joint positions from different Kinects, we use a distance constraint totalPAJ maximization approach. The set of joints deemed to be accurate, i.e. $P A J>0$, are selected and then grouped based on their individual tags. Then starting from the hip center, each group of joints are combined using the Algorithm 1 with the next group selected based on the hierarchy of the joints in the skeleton. The joint state of each joint is determined based on the PAJRatio returned by the algorithm. We used a threshold of 1 for deeming a joint to be tracked correctly by the skeleton combination algorithm. If the root joint, i.e. hip center, is declared not tracked by the algorithm, then the skeleton is invalidated completely - meaning there may not be any person inside the capture area.

The algorithm uses a forward selection based strategy to select the seed point that returns the optimum reward. The reward function is defined as PAJRatio which is just a ratio of the total selected joint position PAJs, and the rejected PAJs. The approach returns the result immediately if the PAJRatio is greater than the PAJ_RATIO_THRESHOLD. We use a high value of 2 for PAJ_RATIO_THRESHOLD, ensuring early termination only if there is a two thirds majority in the selected set. The small number of cameras that actually return good $P A J$ joints enables us to retain a high threshold, without compromising on the run time performance of the algorithm. We use a MAX_DISTANCE_THRESHOLD of $5 \mathrm{~cm}$ to ensure that the joints affected by calibration or fast motion still contribute to the overall joint estimation. By using only the detected joints from various cameras to determine the overall skeleton, it ensures that the approach runs very fast, even for large sets of overlapping cameras.

\section{POINT CLOUD COMBINATION}

Each Kinect gives a depth image, a color image and camera intrinsic values. Using these, the entire captured scene can be projected into 3D space in terms of points. Using the individual Kinect skeleton and a distance based filter we can extract the point cloud data corresponding to the person. These individual point clouds are combined to form one complete and combined 3D point cloud representation of the person. The combination process first downsizes the individual point clouds by a factor of 4 in order to reduce the final point cloud size. These point clouds are then segmented into 25 different segments using their corresponding skeletons. Various approaches exist for human point cloud segmentation. We use a Voronoi based segmentation strategy proposed in [18].

Probability of an Accurate Bone (PAB) is the probability that the skeletal bone segment obtained from the original data is accurate. $\mathrm{PAB}$ of each bone is calculated by taking an average of the PAJ values of the From and the To joint of the corresponding bone. The

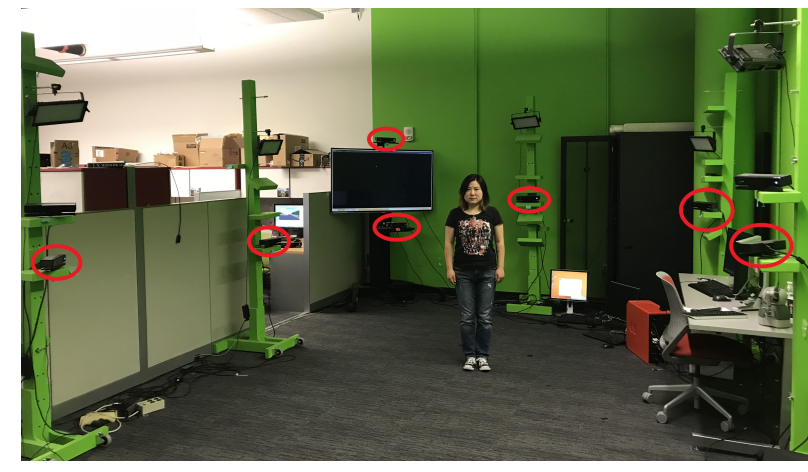

Figure 6: The capture area setup with 7 Kinect cameras marked in red.

inherent Kinect joint estimation is incorporated into the PAJ values and hence only tracked joints and bones are taken into consideration. Following the similar hierarchy as the skeleton combination algorithm 1, we combine the individual point clouds. Applying ICP directly on the entire human point cloud does not consider the inherent problems existing in the individual joints resulting in an inaccurately merged point cloud. Hence, we combine them one segment after another. For any given segment, we start combining the points iteratively by checking if it exists in the combined point cloud or not. If the distance between the new point to be combined and any point already present in the combined point cloud dictionary is less than $1 \mathrm{~mm}$, there is a high probability that both of them correspond to the same point. We take a weighted average of both the points using the PAB value for the particular Kinect skeleton bone, to obtain the new combined point. Using the above mentioned strategy on all the individual segments, we obtain the final combined point cloud which is an accurate 3D model estimation of the real world person.

\section{EVALUATION}

Evaluation of our skeletal pose estimation and point cloud combination method is done on 4 fronts:

- Real-time performance is showcased by calculating the time taken in each step by our methods.

- Objective analysis is performed by calculating the distance based error of the proposed skeleton against the best view Kinect skeleton. We compare our method against 2 state-ofthe-art methods.

- Visual analysis is conducted by checking if the proposed skeleton is contained within the human body. We also show how the visual quality of the combined $3 \mathrm{D}$ point cloud model is improved.

- User performance in an i3DTI game is checked using Holo-Bubble game that we develop.

Video demonstration of the proposed skeletal pose estimation can be found at - https://youtu.be/d0Z9vX5idEA

\subsection{Experimental Setup}

Our i3DTI setup uses 7 Kinect cameras connected to their corresponding machines to capture a large area, where the person can 


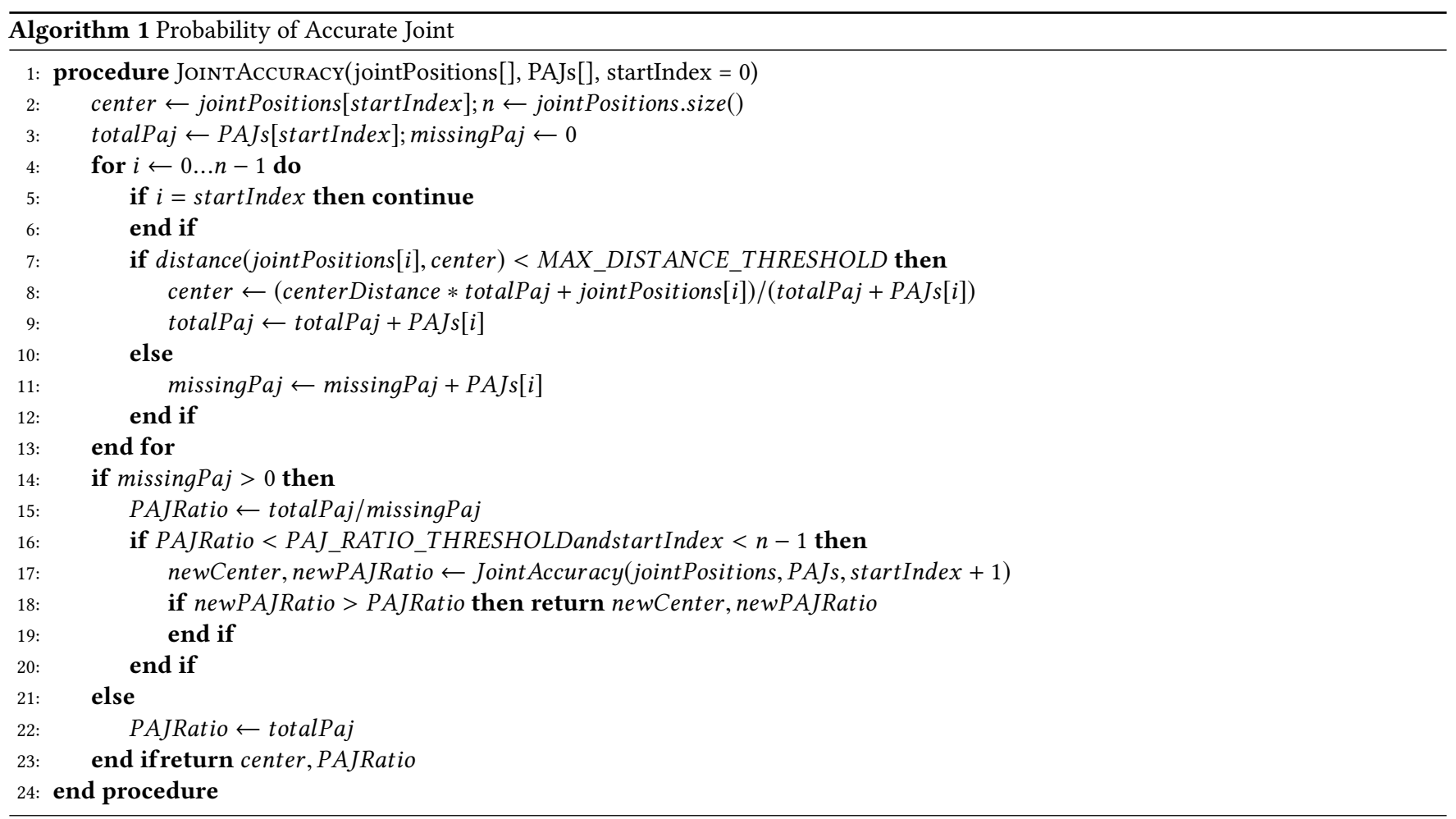

perform different activities. The person was captured in an open scene (15x15sqft space) as shown in Figure 6 . All of the machines were connected by a single gigabit switch. The bulk of the processing was performed on a single estimation machine with Intel i7 2.4GHz processor, 32GB RAM and GTX 970 graphics. All 7 camera machines had Intel Xeon $3.0 \mathrm{GHz}$ processor, 18GB RAM and Nvidia Quadro 4000 graphics. All of the machines were running Windows 8.1 64bit version, and had a Microsoft Kinect V2 connected to it. The code was written in $\mathrm{C}++$ and unmanaged API of the Microsoft Kinect V2 SDK was used. We use the intrinsic calibration of the Kinect, provided by the Microsoft Kinect SDK. Extrinsic calibration is performed to align all the cameras in the same space and to evaluate the accuracy of the point cloud. A planar intersection based calibration approach [2] is used to get the extrinsic calibration between two cameras.

\subsection{Real-time Performance}

Our system is implemented in a distributed manner to achieve faster performance and reduce transmission times. The PAJ calculation for the entire skeleton takes about $1-2 m s$ on an average. All these computations are carried out in the camera machines, and the joint positions along with the PAJ are transmitted to the estimation machine. Due to the small size of the data, it takes less than $1 m s$ to arrive at the estimation machine. The combined skeleton is generated in $1 \mathrm{~ms}$, once all the Kinect machines' data has arrived (about $25 \mathrm{~ms}$ window depending on when the Kinect captures the skeleton). The person's direction estimation, takes $1 \mathrm{~ms}$ if only using the feet or torso direction, $4 m s$ if using depth based knee curvature. So in a live environment, if all the cameras capture at the same time, the proposed skeletal pose estimation strategy is capable of generating a skeleton, even in the worst case, in under $8 \mathrm{~ms}$. The point cloud combination strategy runs over all the points only once and uses dictionary to perform the merging. Hence, the time complexity for the point cloud combination approach is $O(n), n$ being the total number of points from all the point clouds. If there are 7 cameras and each camera captures about 10000 points, it takes only $1-2 m s$ to run the entire point cloud merging task. Both of our methods - skeletal pose estimation and point cloud combination, run in $<10 \mathrm{~ms}$ after the data is obtained from the Kinect cameras, making our entire system work in real time.

\subsection{Objective Analysis}

Majority of the methods in the literature compare their proposed skeleton with the individual Kinect captured skeletons and report the relative distance between them as the accuracy error [13]. [16] manually selects the best view camera frame for comparing their proposed skeleton. [25] compares the bone lengths for their skeleton with the manually set ideal lengths, to evaluate their method. [5] created a dataset that contains manually annotated joint positions. They evaluate their skeleton by finding the average distance error between the estimated skeleton position and ground truth. The above evaluation approaches either annotate the data or use Kinect data as the ground truth. Most of the existing datasets have fixed number of cameras as well as predefined motion while evaluating and hence have not been used for evaluation. However, we evaluate our approach against 2 methods - [13] \& [16].

7 users, 3 male and 4 female, of different heights and sizes were used for the analysis. They were asked to perform 5 sets of motion: 
- Standing in place facing one base camera, without any major movements.

- Standing and rotating in place, to cover the entire scene.

- Walking and rotating in place, to cover the entire scene.

- Roaming around in the entire capture space.

- Free motion in the capture space - bending, crouching, dancing, squats, etc.

Each motion set consisted of around 2000 frames captured from each of the 7 cameras. We use each frame from all the 7 cameras to generate 3 different skeletons, one each using [13], [16] and our method. For each user, we process around 2000 frames per motion * 5 motion sets * 7 cameras $=70000$ skeletons. Hence, for all the 7 users, we process around 500000 skeletons in total.

Distance based error is computed by comparing all the joints of the reference Kinect skeleton against each of the estimated skeletons from the 3 methods - [13], [16] \& our method. We do not need to manually select the reference Kinect skeleton, as done in the other two methods. We calculated the skeleton orientation value $P(S)$ as a part of PAJ, as explained in 4.1. This value determines the probability with which the person is facing each camera. Using this we know which Kinect gives the best view of the person and we use that Kinect's skeleton as the reference.

Table 1 shows the calculated distance based errors. Even though each person was asked to do a specific motion, there is a lot of variation in the way each of them performed. This variation leads to a lot of fluctuation in the computed error scores. Hence for each user, we reported the errors separately for all 5 motions using all the 3 compared methods. Based on the table, it can be seen that the error rates are significantly low for the skeleton estimated by our method, across all users. Thus, our method significantly outperforms the current state-of-the-art methods when objectively comparing the computed distance based error scores.

\subsection{Visual Analysis}

Visual analysis of our system is done on 3 fronts:

- Combined point cloud comparison

- Skeleton containment

- Kinect skeleton comparison

Combined point cloud comparison : Figure 7 shows two different views of the combined point cloud obtained using 7 Kinect cameras. The images in the top row use our point cloud combination strategy. Whereas, the images in the bottom row show the combined point cloud placed together using just the camera calibration without applying our point cloud combination approach. From the Figure 7, it can be seen that our method blends the point clouds well and more accurately. It also gets rid of the common points from multiple cameras, thereby reducing the entire data size. Even at the camera overlapping regions, the number of points are more and the blending is poor if we do not use our point cloud combination strategy.

Skeleton containment : A lot of research has been done to evaluate the accuracy of the Kinect skeleton. Though the Kinect skeleton is stable and accurate to be used in different applications, [27] shows that it is not accurate enough to be considered as ground truth. Thus, just performing comparison with the Kinect skeleton is not a good benchmark. Intuitively, it is known that the skeleton of a
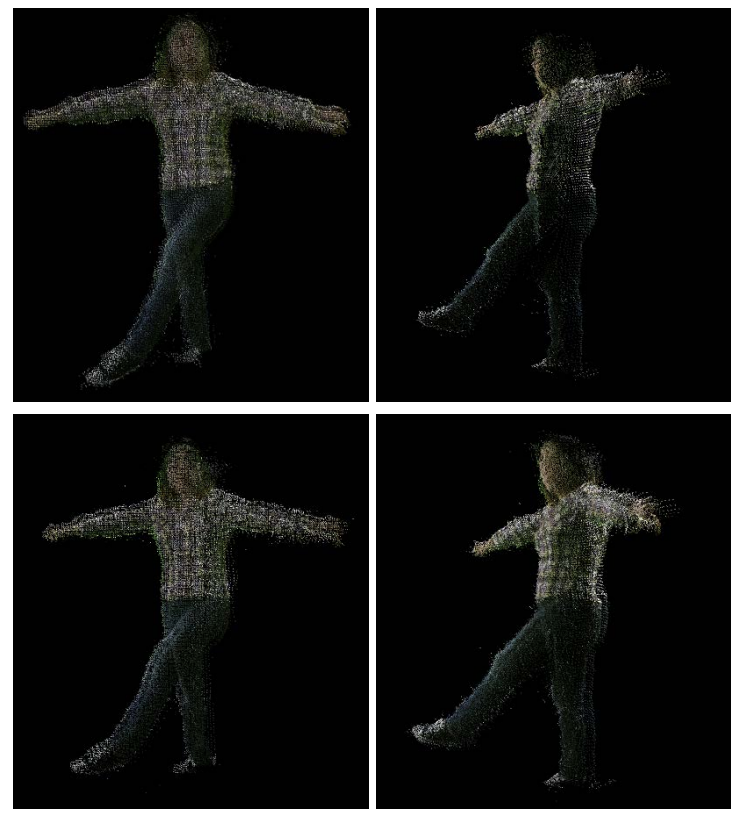

Figure 7: Point cloud images using 7 Kinect cameras showing two different views (one in each column). Top row uses our point cloud combination strategy whereas the bottom row is without using our combination strategy.
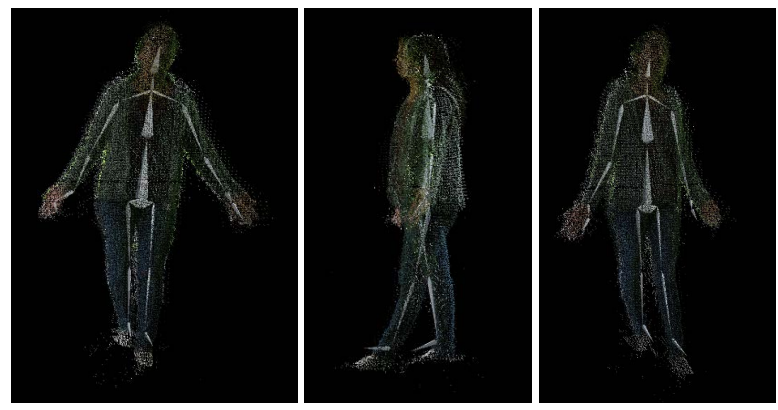

Figure 8: Visual containment check for the combined skeleton and the combined point cloud, from multiple views.

person is contained inside the body. No matter what the angle of the camera is to the person, the skeleton cannot be seen by the camera. This basic fact is used to determine the skeleton correctness. While considering the combined 3D model of the person, all the skeleton joints should be visually contained within the model. Visually we can validate this by placing the combined skeleton on top of the combined point cloud. Figure 8 shows different views of a captured frame, where the $3 \mathrm{D}$ point cloud and the combined skeleton are placed together. It can be visually seen that each and every joint of the proposed skeleton is within the 3D model of the person.

Kinect skeleton comparison : Visually we can check that our method improves the skeletal pose estimation by comparing our skeleton against all the individual Kinect skeletons. Figure 9 shows two different frames captured using two different people. In both the frames, we show the color image from the Kinect and the detected individual skeletons from all 7 Kinect cameras. The last image in the 
Table 1: Distance error (in cm) comparison of our skeleton against 2 methods - [13] \& [16]. 5 motion sets were performed, each set having 2000 skeleton frames from each of the 7 Kinect cameras.

\begin{tabular}{|c|ccc|ccc|cccc|ccc|cc|}
\hline & \multicolumn{3}{|c|}{ Motion-1 } & \multicolumn{3}{c|}{ Motion-2 } & \multicolumn{3}{c|}{ Motion-3 } & \multicolumn{3}{c|}{ Motion-4 } & \multicolumn{3}{c|}{ Motion-5 } \\
\hline User & {$[\mathbf{1 3}]$} & {$[\mathbf{1 6}]$} & Our & {$[\mathbf{1 3}]$} & {$[\mathbf{1 6}]$} & Our & {$[\mathbf{1 3}]$} & {$[\mathbf{1 6}]$} & Our & {$[\mathbf{1 3}]$} & {$[\mathbf{1 6}]$} & Our & {$[\mathbf{1 3}]$} & {$[\mathbf{1 6}]$} & Our \\
\hline 1 & 9.72 & 7.24 & $\mathbf{5 . 4 5}$ & 13.70 & 9.68 & $\mathbf{8 . 3 7}$ & 12.15 & 9.57 & $\mathbf{7 . 9 8}$ & 13.19 & 9.09 & $\mathbf{8 . 4 0}$ & 12.73 & 9.22 & $\mathbf{8 . 6 0}$ \\
\hline 2 & 8.34 & 6.97 & $\mathbf{5 . 3 1}$ & 12.97 & 9.73 & $\mathbf{7 . 9 2}$ & 13.90 & 10.94 & $\mathbf{8 . 6 1}$ & 13.65 & 10.93 & $\mathbf{8 . 7 3}$ & 13.66 & 10.34 & $\mathbf{9 . 0 6}$ \\
\hline 3 & 8.76 & 6.17 & $\mathbf{5 . 1 4}$ & 13.57 & 10.79 & $\mathbf{8 . 3 2}$ & 13.89 & 9.31 & $\mathbf{7 . 5 9}$ & 14.17 & 11.76 & $\mathbf{9 . 5 6}$ & 12.59 & 9.73 & $\mathbf{7 . 7 4}$ \\
\hline 4 & 8.41 & 7.80 & $\mathbf{6 . 1 3}$ & 12.18 & 10.43 & $\mathbf{7 . 3 1}$ & 12.68 & 10.79 & $\mathbf{8 . 3 1}$ & 14.50 & 12.73 & $\mathbf{8 . 9 4}$ & 13.83 & 11.06 & $\mathbf{8 . 4 9}$ \\
\hline 5 & 8.94 & 6.34 & $\mathbf{4 . 7 7}$ & 13.02 & 9.71 & $\mathbf{8 . 8 7}$ & 13.74 & 10.88 & $\mathbf{9 . 6 7}$ & 14.37 & 12.65 & $\mathbf{9 . 8 6}$ & 14.78 & 12.76 & $\mathbf{1 0 . 0 6}$ \\
\hline 6 & 7.36 & 6.73 & $\mathbf{5 . 4 9}$ & 11.97 & 9.36 & $\mathbf{8 . 9 2}$ & 15.67 & 11.67 & $\mathbf{9 . 0 7}$ & 13.73 & 11.37 & $\mathbf{8 . 3 4}$ & 13.17 & 12.94 & $\mathbf{9 . 4 7}$ \\
\hline 7 & 7.95 & 6.16 & $\mathbf{3 . 9 4}$ & 14.61 & 11.69 & $\mathbf{9 . 2 9}$ & 12.95 & 10.94 & $\mathbf{8 . 7 3}$ & 14.91 & 11.97 & $\mathbf{9 . 1 6}$ & 13.90 & 10.73 & $\mathbf{9 . 5 3}$ \\
\hline
\end{tabular}
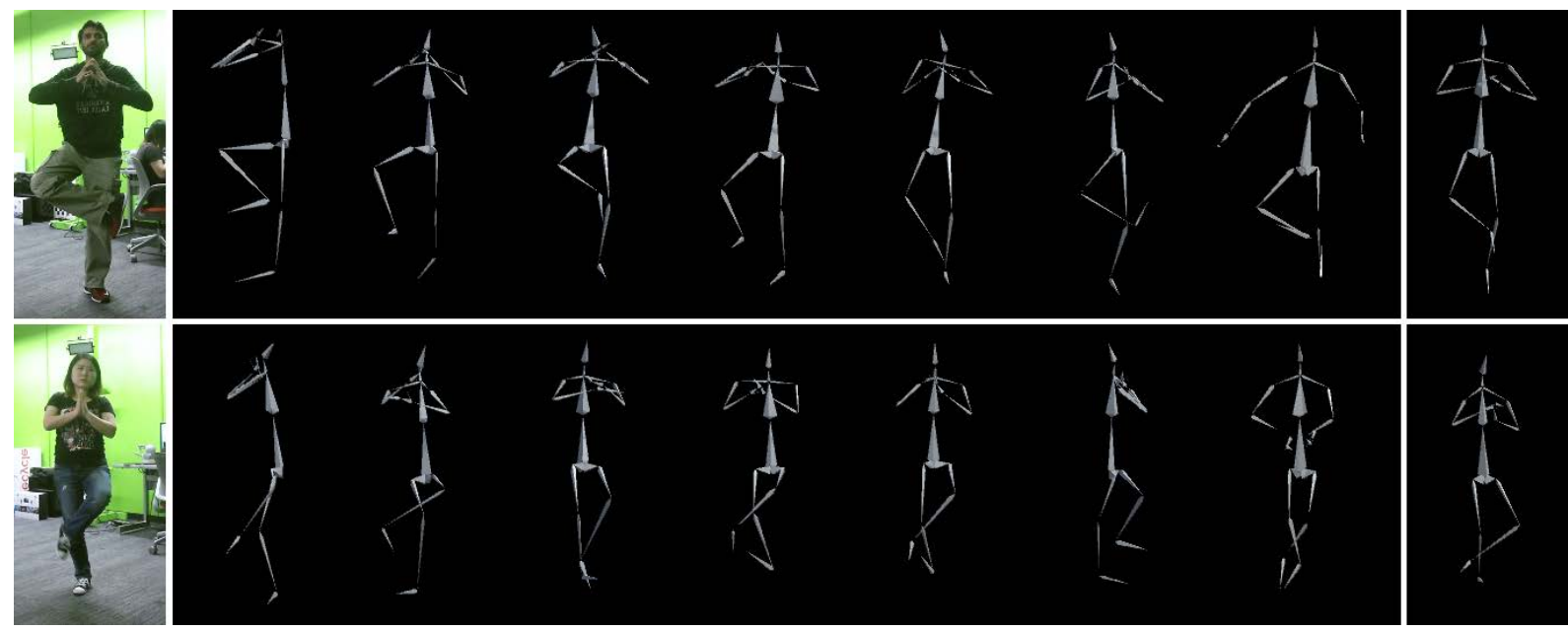

Figure 9: From left to right: Color image, Skeletons from the 7 individual Kinects and our combined Skeleton.

sequence is the combined skeleton estimated from our approach. It can be seen clearly that, most of the Kinect skeletons are inaccurate due to the self-occlusion involved in the person's pose. However, our approach takes care of the self-occlusion issue and is able to estimate a combined skeleton that is an accurate representation of the person's pose. Our method also addresses the problem of occlusion by external objects, as shown in Figure 2 .

\subsection{User Performance in an i3DTI Game}

The objective and visual analyses show how the combined skeleton and point cloud are better than the individual ones. However, it does not show the importance of the proposed approach in terms of its practical usage. Here, we developed an i3DTI game, HoloBubble, which requires the person to move around in the scene to burst the different bubbles popping all around him/her. Figure 10 shows one frame of the Holo-Bubble game where the person is trying to burst the bubbles. The bubbles are randomly created by the 8 generators at predefined speeds. The bubble generators are arranged in a circle, 45 degrees apart, surrounding the user in the center of that circle. Mirrors placed inside the scene allow greater visibility for the players. They are arranged to show the sides of the player, thereby showcasing the entire point cloud. The person interacts with the bubble by bursting it using the colliders attached on the skeleton.

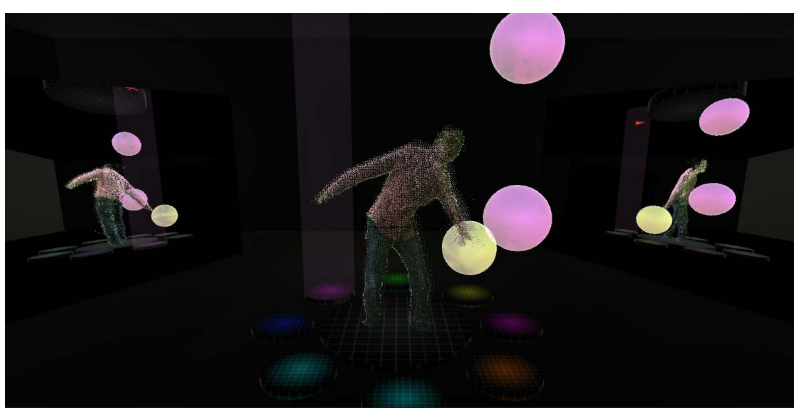

Figure 10: Holo-Bubble frame with the person trying to burst the bubbles, shown as a combined 3D point cloud.

In order to check the user performance, we created two different versions of the game. The Single Camera Version (SCV) used only one Kinect skeleton and point cloud. The Multi Camera Version (MCV) used the combined skeleton and point cloud from 7 Kinect cameras, generated using our approach. Since the bubbles appear all around the user, if only one camera is used to capture the skeleton, the person will not be been able to burst many of the bubbles, eventually not scoring high in the game. We check this hypothesis using a small study we conducted. We check the user's real world 


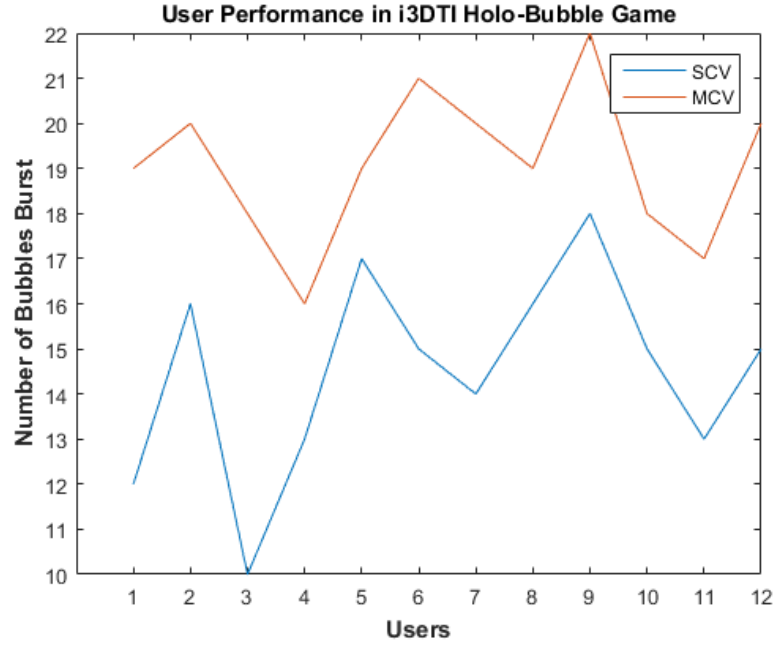

Figure 11: Number of bubbles burst by each user in HoloBubble for Single Camera Version (SCV) and Multi Camera Version (MCV).

performance in both, SCV and MCV. 12 users, 5 male and 7 female were made to play both SCV and MCV in a random order.

Figure 11 shows a graph for the number of bubbles burst, out of 25 , for each user in both versions. As it can be seen from the graph, all the users had better performance of bursting the bubbles in $\mathrm{MCV}$. This shows that our proposed skeleton combination approach can possibly improve user performance in an i3DTI game. We also asked the users which of the two versions was more favorable. All the users agreed that MCV was better. It was clear that the users appreciated the extra effort and the number of cameras put in to improve the skeleton and point cloud.

\subsection{Limitations}

In Section 3 we outlined the different challenges that we face by using a single Kinect skeleton. Some of them, such as motion blur and edge noise are hardware dependent and can only be improved by having better sensors. Regarding other challenges such as occlusion, object interference, jitter and fitting:

- We reduce the jitter significantly by using a temporal smoothing approach [3].

- Using our skeletal pose estimation strategy from multiple Kinects, we reduce the problems occurring from occlusion, object interference and fitting to a great extent.

Even after significant improvement over a single Kinect skeleton approach, the combined skeleton generated using our approach may face some issues. These are mainly due to occlusion of the person from all cameras. In such scenarios, the combined skeleton and point cloud may be incorrect. However, these errors are likely to be transient in nature, depending on the sequence of poses carried out by the users. Since we don't carry forward any information from previous frames for combining skeleton or point cloud, the transient errors do not have impact on quality of subsequent skeleton or point cloud.
Our approach works with the extracted skeletons, making it limited to only Kinect cameras. Kinect was used mainly because of the fact that large number of researchers rely on the use of multiple Kinects for various projects. This dependency on Kinect can easily be removed by using a fast and efficient method to generate a skeleton from the depth frame of any RGB-D camera. The evaluation we have done is same as how the current methods perform, through self-comparison. However, having a motion capture based skeleton will be more accurate and can act as the ground truth for proper evaluation.

\section{CONCLUSION}

In this paper, we present a real time multi-Kinect skeletal pose estimation technique for combined 3D human model generation. Our method is able to identify the skeleton of a person, even when they are in partial view of the Kinect camera. By leveraging the detected skeleton from individual cameras, it is possible to get an accurate estimation of the person's pose without the need to learn large amounts of pose data. Our method first calculates PAJ for all joints and all skeletons, to indicate the likelihood that a particular joint is estimated correctly. A greedy distance constraint algorithm combines the joints from different camera views providing accurate estimations for even partially visible joints. We also introduce probability of accurate bone, $\mathrm{PAB}$, using the calculated PAJ values. We use it to merge the point clouds from individual cameras to a combined point cloud, one segment after another. Skeleton correctness measures are also proposed to ensure that the skeleton is inside the person and of the correct size. Distance based error scoring is used to compare our method against other approaches. Visual analysis is performed by showing that the estimated skeleton is always contained within the human model. A 3D Holo-Bubble game is generated to showcase the real-time performance of our combined skeleton and point cloud. Based on objective, visual and performance analysis, our method performed better than the other state-of-the-art methods using multiple RGB-D cameras, without needing any training or large data sets.

\section{ACKNOWLEDGMENTS}

This material is based upon work supported by the National Science Foundation under Grant No. 1012975. Any opinions, findings, conclusions or recommendations expressed in this material are those of author(s) and do not necessarily reflect the views of the National Science Foundation.

\section{REFERENCES}

[1] S. Asteriadis, A. Chatzitofis, D. Zarpalas, D. S. Alexiadis, and P. Daras. Estimating human motion from multiple kinect sensors. In Proceedings of the 6th International Conference on Computer Vision / Computer Graphics Collaboration Techniques and Applications, MIRAGE '13, pages 3:1-3:6, New York, NY, USA, 2013. ACM.

[2] E. Auvinet, J. Meunier, and F. Multon. Multiple depth cameras calibration and body volume reconstruction for gait analysis. In Information Science, Signal Processing and their Applications (ISSPA), 2012 11th International Conference on, pages 478-483, July 2012.

[3] M. Azimi. Skeletal joint smoothing white paper. MSDN digital library, 2012.

[4] N. A. Azis, Y. S. Jeong, H. J. Choi, and Y. Iraqi. Weighted averaging fusion for multi-view skeletal data and its application in action recognition. IET Computer Vision, 10(2):134-142, 2016.

[5] M. Carraro, M. Munaro, A. Roitberg, and E. Menegatti. Improved Skeleton Estimation by Means of Depth Data Fusion from Multiple Depth Cameras, pages 1155-1167. Springer International Publishing, Cham, 2017. 
[6] L. Cruz, D. Lucio, and L. Velho. Kinect and rgbd images: Challenges and applications. In Graphics, Patterns and Images Tutorials (SIBGRAPI-T), 2012 25th SIBGRAPI Conference on, pages 36-49, Aug 2012.

[7] K. Desai, K. Bahirat, S. Ramalingam, B. Prabhakaran, T. Annaswamy, and U. E. Makris. Augmented reality-based exergames for rehabilitation. In Proceedings of the 7th International Conference on Multimedia Systems, MMSys '16, pages 22:1-22:10, 2016.

[8] K. Desai, U. H. H. Belmonte, R. Jin, B. Prabhakaran, P. Diehl, V. A. Ramirez, V. Johnson, and M. Gans. Experiences with multi-modal collaborative virtual laboratory (mmcvl). In 2017 IEEE Third International Conference on Multimedia Big Data (BigMM), pages 376-383, April 2017.

[9] K. Desai, S. Raghuraman, R. Jin, and B. Prabhakaran. Qoe studies on interactive 3d tele-immersion. In 2017 IEEE International Symposium on Multimedia (ISM), Dec 2017.

[10] M. Dou, S. Khamis, Y. Degtyarev, P. Davidson, S. R. Fanello, A. Kowdle, S. O. Escolano, C. Rhemann, D. Kim, J. Taylor, P. Kohli, V. Tankovich, and S. Izadi. Fusion4d: Real-time performance capture of challenging scenes. ACM Trans. Graph., 35(4):114:1-114:13, July 2016.

[11] E. Haller, G. Scarlat, I. Mocanu, and M. Trăscău. Human Activity Recognition Based on Multiple Kinects, pages 48-59. Springer Berlin Heidelberg, Berlin, Heidelberg, 2013.

[12] S. Izadi, D. Kim, O. Hilliges, D. Molyneaux, R. Newcombe, P. Kohli, J. Shotton, S. Hodges, D. Freeman, A. Davison, and A. Fitzgibbon. Kinectfusion: Real-time $3 \mathrm{~d}$ reconstruction and interaction using a moving depth camera. In Proceedings of the 24th Annual ACM Symposium on User Interface Software and Technology, UIST '11, pages 559-568, New York, NY, USA, 2011. ACM.

[13] S. Kaenchan, P. Mongkolnam, B. Watanapa, and S. Sathienpong. Automatic multiple kinect cameras setting for simple walking posture analysis. In 2013 International Computer Science and Engineering Conference (ICSEC), pages 245249, Sept 2013.

[14] J.-M. Lien, G. Kurillo, and R. Bajcsy. Multi-camera tele-immersion system with real-time model driven data compression: A new model-based compression method for massive dynamic point data. Vis. Comput., 26(1):3-15, Nov. 2009.

[15] Microsoft. Kinect SDK. www.microsoft.com/en-us/kinectforwindows/âĂÖ, March 2013.

[16] J. C. Núñez, R. Cabido, A. S. Montemayor, and J. J. Pantrigo. Real-time human body tracking based on data fusion from multiple rgb-d sensors. Multimedia Tools Appl., 76(3):4249-4271, Feb. 2017.

[17] S. Raghuraman, K. Bahirat, and B. Prabhakaran. Evaluating the efficacy of rgb-d cameras for surveillance. In 2015 IEEE International Conference on Multimedia and Expo (ICME), pages 1-6, June 2015.

[18] S. Raghuraman, K. Venkatraman, Z. Wang, B. Prabhakaran, and X. Guo. A 3d tele-immersion streaming approach using skeleton-based prediction. In $A C M$ Multimedia, pages 721-724, 2013.

[19] E. Rosten and T. Drummond. Machine learning for high-speed corner detection In European Conference on Computer Vision, volume 1, pages 430-443, May 2006.

[20] S. Rusinkiewicz and M. Levoy. Efficient variants of the icp algorithm. In Proceedings Third International Conference on 3-D Digital Imaging and Modeling, pages 145-152, 2001.

[21] J. Shotton, A. Fitzgibbon, M. Cook, T. Sharp, M. Finocchio, R. Moore, A. Kipman, and A. Blake. Real-time human pose recognition in parts from single depth images. In R. Cipolla, S. Battiato, and G. M. Farinella, editors, Machine Learning for Computer Vision, volume 411 of Studies in Computational Intelligence, pages 119-135. Springer Berlin Heidelberg, 2013.

[22] L. Shuai, C. Li, X. Guo, B. Prabhakaran, and J. Chai. Motion capture with ellipsoidal skeleton using multiple depth cameras. IEEE Transactions on Visualization and Computer Graphics, 23(2):1085-1098, Feb 2017.

[23] Y. Tian, S. Raghuraman, T. Annaswamy, A. Borresen, K. Nahrstedt, and B. Prabhakaran. H-time: Haptic-enabled tele-immersive musculoskeletal examination. 2017

[24] L. Yang, L. Zhang, H. Dong, A. Alelaiwi, and A. El Saddik. Evaluating and improving the depth accuracy of kinect for windows v2. Sensors fournal, IEEE, 15(8):4275-4285, Aug 2015.

[25] K.-Y. Yeung, T.-H. Kwok, and C. C. Wang. Improved skeleton tracking by duplex kinects: A practical approach for real-time applications. fournal of Computing and Information Science in Engineering, 13(4):041007, 2013.

[26] L. Zhang, J. Sturm, D. Cremers, and D. Lee. Real-time human motion tracking using multiple depth cameras. In 2012 IEEE/RS7 International Conference on Intelligent Robots and Systems, pages 2389-2395, Oct 2012.

[27] L. Zhou, Z. Liu, H. Leung, and H. P. H. Shum. Posture reconstruction using kinect with a probabilistic model. In Proceedings of the 20th ACM Symposium on Virtual Reality Software and Technology, VRST '14, pages 117-125, New York, NY, USA 2014. ACM. 